\title{
The effect of nicardipine on surgical pleth index during thyroidectomy under desflurane anaesthesia: a prospective randomized controlled trial
}

Byung Gun Lim, Young Ju Won, Gwi Eun Yeo, Sohyun Lee, Chunghun Lee and II Ok Lee

Department of Anaesthesiology and Pain Medicine, Korea University Guro Hospital, Seoul, Korea, Republic of

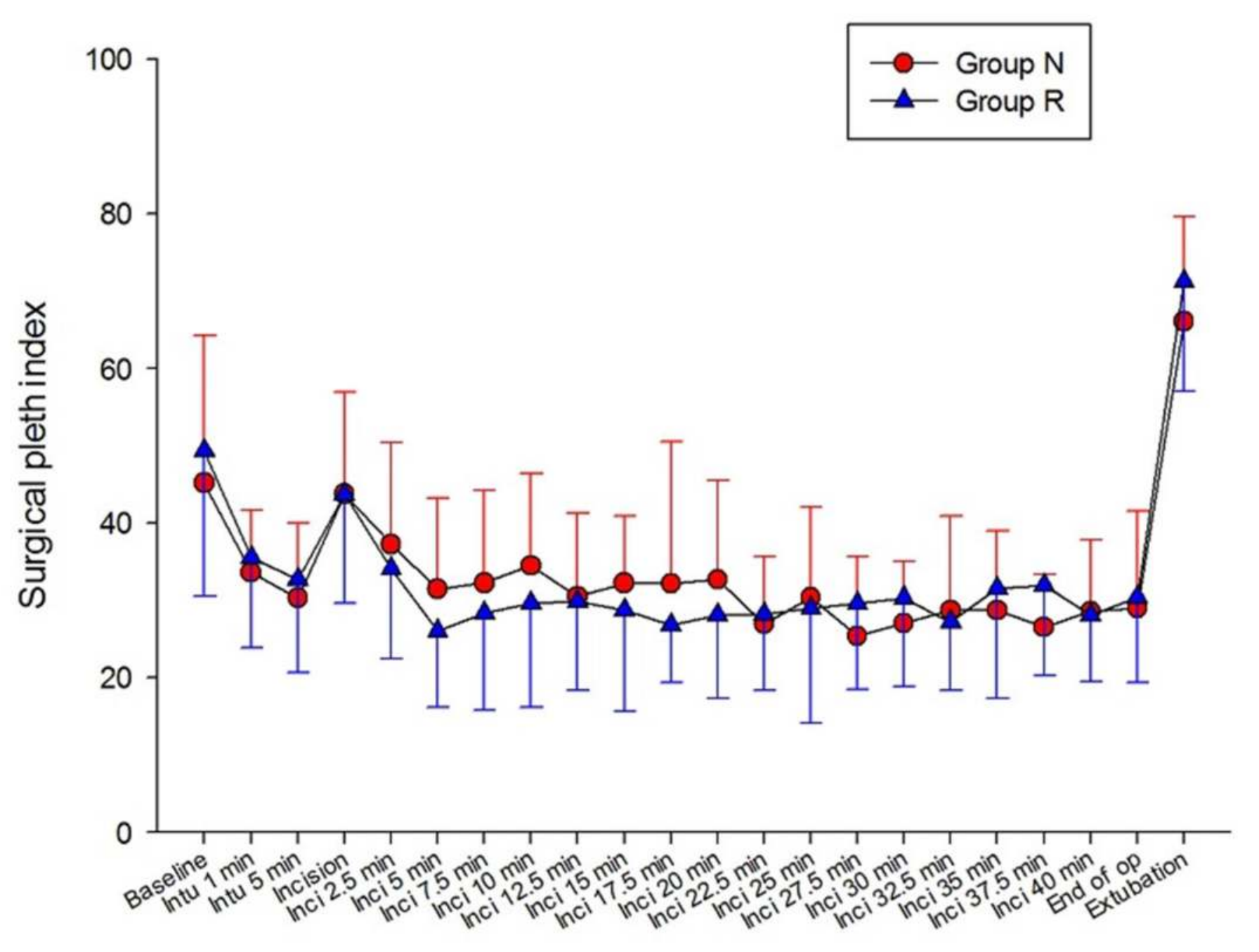

Time point

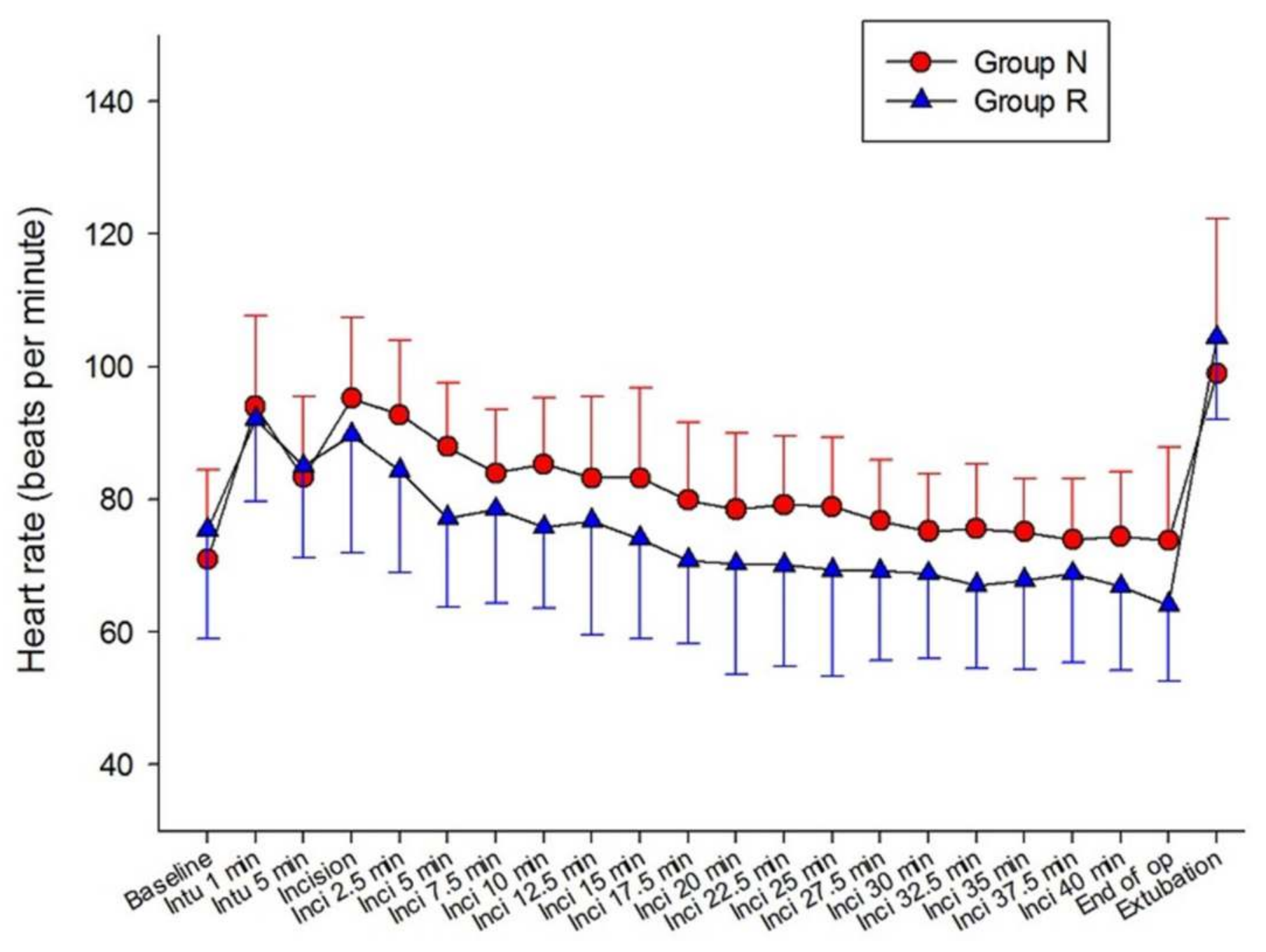

Time point

Fig. 1. Trend of surgical pleth index (left) and heart rate (right) in patients receiving nicardipine or remifentanil during anaesthesia and surgery.

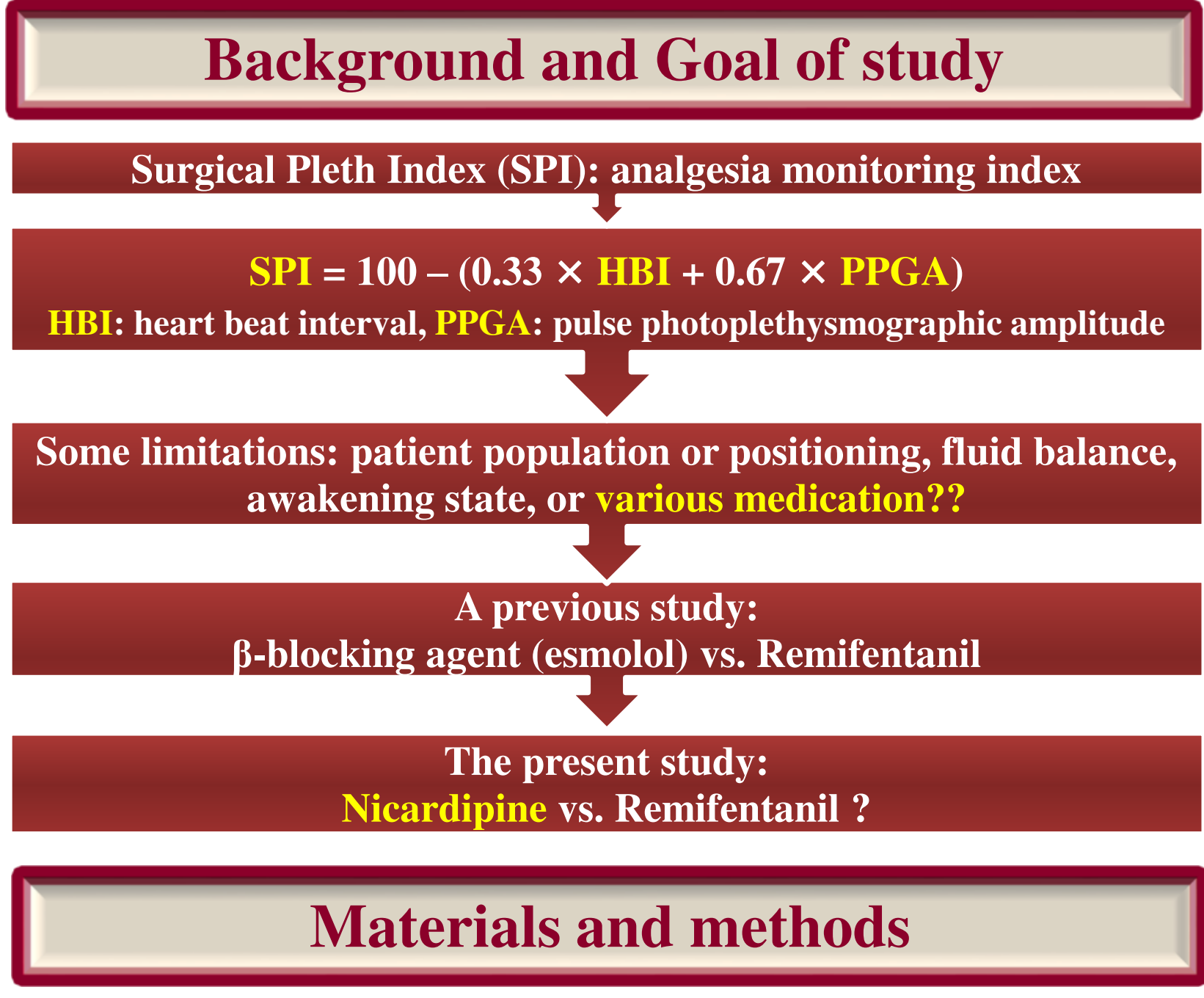

- Prospective, randomized controlled, single center study

- Subjects: 40 patients; 20-65 years, ASA physical status I $\sim I$ undergoing thyroidectomy

- Group assignment: nicardipine (Group N), remifentanil (Group R) according to infusion drug

- Premedication: midazolam $2 \mathrm{mg}$, iv

- Induction: propofol $2 \mathrm{mg} / \mathrm{kg}$, fentanyl $1 \mu \mathrm{g} / \mathrm{kg}$ and rocuronium 0.6 $\mathrm{mg} / \mathrm{kg}$

- Maintenance: desflurane + nitrous oxide $50 \%$ in oxygen

- Infusion (after intubation): nicardipine or remifentanil

- Target: Mean blood pressure (MBP) within $\pm 20 \%$ from the preoperative value

- Dosing: nicardipine - $100 \mu \mathrm{g}$ bolus followed by $0.5 \mu \mathrm{g} / \mathrm{kg} / \mathrm{min}$; remifentanil - $50 \mu \mathrm{g}$ bolus followed by $0.05 \mu \mathrm{g} / \mathrm{kg} / \mathrm{min} \rightarrow$ titration at every $2.5 \mathrm{~min}$ interval

\begin{tabular}{|c|c|}
\hline & Assessment \\
\hline OR & $\begin{array}{ll}\checkmark & \text { SPI } \\
\checkmark & \text { BIS } \\
\checkmark & \text { Et Des } \\
\checkmark & \text { MBP } \\
\checkmark & \text { Heart rate } \\
\checkmark & \text { Extubation time }\end{array}$ \\
\hline PACU & $\begin{array}{l}\text { Recovery time } \\
\text { (time to reach a modified Aldrete score of 10) } \\
\text { Adverse events }\end{array}$ \\
\hline
\end{tabular}

\section{Results and discussion}

- There was no significant difference between the groups in patients' characteristics (Table 1).

- The trend of SPI value was comparable between the groups during surgery $(\mathrm{P}=0.76)$, although the trend of heart rate was significantly different between the groups $(\mathrm{P}=0.03$; HR was generally higher in group $\mathrm{N}$ than in group $\mathrm{R}$ ) (Figure 1: primary outcome).

- The trends of intraoperative BIS, end-tidal desflurane concentration (Et Des) and MBP were comparable between the groups.

- Extubation time, recovery time and incidence of nausea/vomiting at PACU were comparable between the groups (Table 2).

Table 1. Demographic and clinical data

\begin{tabular}{|c|c|c|}
\hline $\begin{array}{c}\text { Values are mean } \pm \text { SD, median (range) or } \\
\text { number of patients. }\end{array}$ & $\begin{array}{r}\text { Group N } \\
(n=19)\end{array}$ & $\begin{array}{c}\text { Group R } \\
(n=21)\end{array}$ \\
\hline Age (yr) & $46(26-60)$ & $46(34-68)$ \\
\hline $\operatorname{Sex}(M / F)$ & $3 / 16$ & $4 / 17$ \\
\hline Body mass index $\left(\mathbf{k g} / \mathbf{m}^{2}\right)$ & $24.5 \pm 2.6$ & $23.6 \pm 2.5$ \\
\hline Anaesthesia time (min) & $97.4 \pm 26.0$ & $101.9 \pm 20.4$ \\
\hline Surgical time (min) & $69.6 \pm 23.8$ & $70.5 \pm 18.2$ \\
\hline Intraoperative fluid (ml/kg) & $4.1 \pm 2.5$ & $3.7 \pm 1.5$ \\
\hline Baseline SPI value & $45.2 \pm 19.1$ & $49.4 \pm 18.9$ \\
\hline Baseline mean arterial pressure $(\mathrm{mmHg})$ & $92.7 \pm 10.6$ & $93.3 \pm 7.8$ \\
\hline Baseline heart rate (beats per min) & $71.0 \pm 13.6$ & $75.4 \pm 16.4$ \\
\hline
\end{tabular}

Table 2. Recovery profiles and perioperative medicine

\begin{tabular}{lccc}
\hline $\begin{array}{l}\text { Values are mean } \pm \text { SD or number of } \\
\text { patients. }\end{array}$ & $\begin{array}{c}\text { Group N } \\
(n=19)\end{array}$ & $\begin{array}{c}\text { Group R } \\
(n=21)\end{array}$ & $P$ value \\
\hline Extubation time (sec) & $419.8 \pm 105.0$ & $416.5 \pm 161.6$ & 0.94 \\
Recovery time $(\mathrm{min})$ & $23.7 \pm 9.0$ & $26.2 \pm 10.7$ & 0.43 \\
Infused rate of nicardipine $(\mu \mathrm{g} / \mathrm{kg} / \mathrm{min})$ & $0.57 \pm 0.09$ & & \\
Infused rate of remifentanil $(\mu \mathrm{g} / \mathrm{kg} / \mathrm{min})$ & & $0.05 \pm 0.01$ & \\
Nausea or vomiting at PACU $(\mathrm{n})$ & $4 / 19$ & $3 / 21$ & 0.57 \\
\hline
\end{tabular}

\section{Conclusions}

- The administration of nicardipine may confound the interpretation of SPI as a surrogate measure of the nociception-antinociception balance during general anaesthesia.

- SPI does not seem to reflect the level of surgical stress and may not help guide the use of opioids (analgesics) in the clinical settings with the administration of nicardipine during general anaesthesia.

* This report was supported by a Korea University Guro Hospital Grant (Grant number: O1400051) awarded to Dr. Byung Gun Lim from Korea University Guro Hospital (Seoul, Korea, Republic of). 